TRANSACTIONS OF THE

AMERICAN MATHEMATICAL SOCIETY

Volume 354, Number 7, Pages 2707-2724

S 0002-9947(02)02996-3

Article electronically published on February 25, 2002

\title{
SELF-TILTING COMPLEXES YIELD UNSTABLE MODULES
}

\author{
ALEXANDER ZIMMERMANN
}

\begin{abstract}
Let $G$ be a group and $R$ a commutative ring. Let $\operatorname{TrPic} R(R G)$ be the group of isomorphism classes of standard self-equivalences of the derived category of bounded complexes of $R G$-modules. The subgroup $H D_{R}(G)$ of $\operatorname{TrPic}_{R}(R G)$ consisting of self-equivalences fixing the trivial $R G$-module acts on the cohomology ring $H^{*}(G, R)$. The action is functorial with respect to $R$. The self-equivalences which are 'splendid' in a sense defined by J. Rickard act naturally with respect to transfer and restriction to centralizers of $p$-subgroups in case $R$ is a field of characteristic $p$. In the present paper we prove that this action of self-equivalences on $H^{*}(G, R)$ commutes with the action of the Steenrod algebra, and study the behaviour of the action of splendid self-equivalences with respect to Lannes' $T$-functor.
\end{abstract}

\section{INTRODUCTION}

In an earlier joint paper [9] with Raphaël Rouquier I defined $\operatorname{Tr} P i c_{R}(A)$, the group of standard self-equivalences of a derived module category $D^{b}(A)$ for an $R$ algebra $A$ which is projective as an $R$-module. For any $A$-module $M$ let $H D_{M}(A)$ be the subgroup of $\operatorname{TrPic} c_{R}(A)$ which is formed by the self-equivalences mapping $M$ to an isomorphic copy. Then, in an earlier paper [11] I showed that, under a certain hypothesis on $M$, the group $H D_{M}(A)$ acts in a natural way on the Extalgebra $\operatorname{Ext}_{A}^{*}(M, M)$. When $A$ is a group algebra $R G$, with $R$ being a field of characteristic $p$ and $G$ being a finite group, J. Rickard defined in [8] a splendid equivalence by some technical conditions, basically by asking that the homogeneous components of a tilting complex be $p$-permutation modules induced from diagonal $p$-subgroups, and by an invertibility condition in the homotopy category. These splendid equivalences induce self-equivalences of the derived categories of centralizers of $p$-subgroups by the Brauer construction. In [12] I showed that then, for $M=R$ being the trivial module, the actions of those splendid equivalences commute with transfer and restriction from and to the cohomology rings of centralizers of $p$-subgroups. In the present paper we enlarge these properties still further. The action of self-equivalences of the bounded derived category $D^{b}(R G)$ on $H^{*}(G, R)$ commutes with the action of the Steenrod algebra on $H^{*}(G, R)$ for any prime $p$.

As a consequence of the above statements, any cohomology ring $H^{*}\left(G, \mathbb{F}_{p}\right)$ defines a functor $H^{*}\left(G, \mathbb{F}_{p}\right) \otimes_{\mathbb{F}_{p} H D_{\mathbb{F}_{p}}\left(B_{0}\left(\mathbb{F}_{p} G\right)\right)}$ - from the modules over the group of derived self-equivalences of the principal block of the group ring $\mathbb{F}_{p} H D_{\mathbb{F}_{p}}\left(\mathbb{F}_{p} G\right)$ fixing the trivial module to the category of unstable modules $\mathfrak{U}_{p}$ and similarly in the opposite direction. Obviously, we may restrict to splendid self-equivalences.

Received by the editors August 28, 2001.

2000 Mathematics Subject Classification. Primary 16E30, 20J06, 55S10, 18E30. 
We shall describe the composition of Lannes' $T$-functor with the first functor and the image of free unstable modules by the second. Moreover, by a result of Lannes $T_{V}\left(H^{*}\left(G, \mathbb{F}_{p}\right)\right)$ decomposes into a direct product of cohomology rings as unstable modules. We shall prove that this decomposition is also a decomposition as modules over the action of splendid self-equivalences. This will give evidence that splendid equivalences are the correct objects to study in this context.

The paper is organized as follows. Section 1 recalls the necessary definitions and properties of Evens' norm map as it is used here and the definition of the Steenrod operation. In Section 2 it is shown that the normalized part of the outer automorphism group of the group ring behaves well with respect to the norm map norm ${ }_{G}^{G \times C_{p}}$ on the cohomology ring of $G$. Section 3 proves that splendid self-equivalences of the derived category of a group ring fixing the trivial module act equivariantly with respect to the Steenrod operation on the cohomology ring of the group. Finally, in Section 4 we work on the relation of unstable modules and the action of splendid self-equivalences of the derived category of a group ring.

Acknowledgement. I thank Serge Bouc for many fruitful discussions over the last years. In particular he mentioned to me a subtlety concerning the difference between homotopy equivalences and quasi-isomorphisms for tensor product complexes which led to Remark 3.4. I also thank the referee for pointing out an error in the proof of Proposition 2.1 in the original version of the paper.

\section{Preliminary definitions And KnOWn Results}

1.1. Equivalences between derived categories of group rings. The notation concerning derived categories and derived equivalences we use here and throughout the whole article is the one in [5]. Let $R$ be a commutative ring and let $A$ and $B$ be $R$-algebras. A complex $T$ is a tilting complex if it is a bounded complex with finitely generated projective homogeneous components so that $\operatorname{Hom}_{D^{b}(A)}(T, T[n])=0$ for $n \neq 0$ and so that the smallest triangulated subcategory of $D^{b}(A)$ which is closed under taking direct summands of finite direct sums and which contains $T$ also contains the rank 1 free module $A$. The fundamental result of Rickard [7] shows that the derived categories $D^{b}(A)$ and $D^{b}(B)$ are equivalent as triangulated categories if and only if there is a tilting complex $T$ in $D^{b}(A)$ so that $\operatorname{End}_{D^{b}(A)}(T) \simeq B$.

If $A$ is flat as an $R$-module, then for any tilting complex $T$ there is a complex $X$ in $D^{b}\left(A \otimes_{R} B^{o p}\right)$ so that $X \otimes_{B}^{\mathbb{L}}-$ is an equivalence which maps $B$ to $T$. A complex $X$ in $D^{b}\left(A \otimes_{R} B^{o p}\right)$ so that $X \otimes_{B}^{\mathbb{L}}$ - is an equivalence is called a two-sided tilting complex. Suppose $A=B$ and $A$ is projective as an $R$-module. Then (cf. [9]), the set $\operatorname{TrPic} c_{R}(A)$ of isomorphism classes of two-sided tilting complexes in $D^{b}\left(A \otimes_{R} A^{o p}\right)$ is a group with group law $-\otimes_{A}^{\mathbb{L}}-$.

For any $A$-module $M$ set

$$
H D_{M}(A):=\left\{[X] \in \operatorname{TrPic} R(A) \mid X \otimes_{A}^{\mathbb{L}} M \simeq M\right\} .
$$

If any automorphism of $M$ is given by multiplication with a unit in the centre of $A$, then in [11] it is shown that $\operatorname{Ext}_{A}^{*}(M, M)$ is an $R H D_{M}(A)$-module by the action

$$
\begin{aligned}
& H_{D^{b}(A)}(M, M[n]) \longrightarrow H_{D^{b}(A)}\left(X \otimes_{A}^{\mathbb{L}} M, X \otimes_{A}^{\mathbb{L}} M[n]\right) \\
& \left(\alpha_{X}^{-1}\right)^{*}\left(\alpha_{X}\right)_{*} \operatorname{Hom}_{D^{b}(A)}(M, M[n])
\end{aligned}
$$

where $\alpha_{X}: X \otimes_{A}^{\mathbb{L}} M \longrightarrow M$ is an isomorphism. 
In case $A=R G$ is a group ring for a finite group $G$, a more subtle notion is useful. Let $R$ be a field of characteristic $p$ or a complete discrete valuation ring of characteristic 0 with residue field of characteristic $p$. Let $B$ be the principal block of $R G$ and let $P$ be a Sylow $p$-subgroup of $G$. Let $\Delta$ be the diagonal embedding of $G$ into $G \times G$. A two-sided tilting complex $X$ in $D^{b}\left(B \otimes B^{o p}\right)$ is a splendid complex (cf. Rickard [8]) if $X$ is a complex of modules whose homogeneous components are projective as right $R G$-modules and projective as left $R G$-modules, so that $\operatorname{Hom}_{R G}^{\bullet}(X, X)$ is homotopy equivalent to $B$ as a complex of left and as a complex of right modules, and so that each of the homogeneous components of $X$ are $\Delta(P)$-projective $p$-permutation modules. Let $\operatorname{SplenPic}_{R}(G)$ be the set of homotopy equivalence classes $(X)$ of splendid complexes $X$ whose isomorphism class is in $\operatorname{TrPic}_{R}(B)$. Let

$$
H_{S^{\prime} p l e n}(G):=\left\{(X) \in \operatorname{SplenPic}_{R}(G) \mid[X] \in H D_{R}(B)\right\} .
$$

Suppose that $R$ is a field of characteristic $p$. Then, for any $p$-subgroup $Q$ of $G$, denote by $b$ the principal block of $R C_{G}(Q)$. The Brauer functor

$$
B \otimes B^{o p}-\bmod \ni N \mapsto N(\Delta Q):=N^{\Delta Q} / \sum_{S<Q ; S \neq Q} N^{\Delta S} \in b \otimes b^{o p}-\bmod
$$

induces a homomorphism

$$
\operatorname{SplenPic}_{R}(G) \longrightarrow \operatorname{SplenPic}_{R}\left(C_{G}(Q)\right) .
$$

In [12] it is shown that the action of an $X$ in $\operatorname{HSplen}_{R}(G)$ so that $X(\Delta(Q)) \in$ $H_{S^{\prime} p l e n_{R}}\left(C_{G}(Q)\right)$ on $H^{*}(G, R)$ commutes with restriction $\operatorname{res}_{C_{G}(Q)}^{G}$ and transfer $\operatorname{tr}_{C_{G}(Q)}^{G}$ between $H^{*}(G, R)$ and $H^{*}\left(C_{G}(Q), R\right)$.

1.2. Steenrod operations. Let $G$ be a group and let $H$ be a subgroup of $G$ of finite index. Then, $G \leq H<\mathfrak{S}_{|G: H|}$ via the action of $G$ on the cosets modulo $H$. Suppose $R$ is a commutative, hereditary coefficient ring.

We recall below the definitions of Evens' norm map and Steenrod operations from Benson's book 1 .

Let $\mathbf{P}=\left(P_{i}, d_{i}\right)_{i \in \mathbb{N}}$ be a projective resolution of $R$ as $R H$-module. Then, $\bigotimes_{i=1}^{|G: H|} \mathbf{P}$ is a (not necessarily projective) resolution of $R$ as $R\left(H \imath \mathfrak{S}_{|G: H|}\right.$ )-module. Let $\mathbf{Q}=\left(Q_{i}, \partial_{i}\right)_{i \in \mathbb{N}}$ be a projective resolution of $R$ as $R\left(H \imath \mathfrak{S}_{|G: H|}\right)$-module. Then, there is a quasiisomorphism of $R\left(H \imath \mathfrak{S}_{|G: H|}\right)$-module complexes $\tau: \mathbf{Q} \longrightarrow \bigotimes_{i=1}^{|G: H|} \mathbf{P}$.

We denote by $R_{s}$ the sign representation of $\mathfrak{S}_{|G: H|}$, and by $R_{s}^{(r)}$ the $r$-fold tensor product of $R_{s}$ with itself.

An element $x \in H^{n}(H, M)$ is given as an equivalence class of homomorphisms with representative $x: P_{n} \longrightarrow M$, and this defines a homomorphism $\bigotimes_{i=1}^{|G: H|} P_{n} \longrightarrow$ $\bigotimes_{i=1}^{|G: H|} M$ of $R\left(H<\mathfrak{S}_{|G: H|}\right)$-modules. As $\bigotimes_{i=1}^{|G: H|} P_{n}$ is a direct factor of the degree $|G: H| \cdot n$-homogeneous component of $\bigotimes_{i=1}^{|G: H|} \mathbf{P}$, the cocycle $x$ defines a homomorphism

$$
Q_{n \cdot|G: H|} \longrightarrow\left(\bigotimes_{i=1}^{|G: H|} \mathbf{P}\right)_{n \cdot|G: H|} \longrightarrow \bigotimes_{i=1}^{|G: H|} P_{n} \longrightarrow \bigotimes_{i=1}^{|G: H|} M \otimes R_{s}^{(n)}
$$

We have to tensor with $R_{s}^{(n)}$ in order to get the mapping well defined with respect to the sign convention for tensor products of complexes. Observe that for even degree cohomology this is the tensor product with the identity. The restriction to 
$G \subseteq\left(H \prec \mathfrak{S}_{|G: H|}\right)$ defines an element $\operatorname{norm}_{H}^{G}(x)$ in $H^{n|G: H|}\left(G, \bigotimes_{i=1}^{|G: H|} M \otimes_{R} R_{s}^{(n)}\right)$. However, the sign of $\operatorname{norm}_{H}^{G}(x)$ depends on the embedding of $G$ into $H$ 々 $\mathfrak{S}_{|G: H|}$. Since later on the embedding will be fixed once and for all, this ambiguity will not be of importance for us.

The Steenrod operations are defined by the following procedure.

Observe that, when $p$ is odd, any $p$-cycle is of even parity, and so one can forget the sign representation which had to be introduced earlier in order to get the norm map well defined. We take

$$
\operatorname{norm}_{G}^{G \times C_{p}}: H^{n}\left(G, \mathbb{F}_{p}\right) \longrightarrow H^{n \cdot p}\left(G \times C_{p}, \mathbb{F}_{p}\right) \simeq \bigoplus_{i=0}^{n p} H^{n p-i}\left(G, \mathbb{F}_{p}\right) \otimes_{\mathbb{F}_{p}} H^{i}\left(C_{p}, \mathbb{F}_{p}\right)
$$

and express

$$
\operatorname{norm}_{G}^{G \times C_{p}}(x)=\bigoplus_{i=0}^{n p} D^{n p-i}(x) \otimes a_{i},
$$

where $a_{2 i}=a_{1}^{2 i}$ and $a_{2 i+1}=\beta\left(a_{1}\right) a_{2 i}$. We define

$$
S q^{i}:=D^{n-i}: H^{n}\left(G, \mathbb{F}_{2}\right) \longrightarrow H^{n+i}\left(G, \mathbb{F}_{2}\right)
$$

if $p=2$ and

$$
P^{i}:=(-1)^{i+m n(n+1) / 2}(m !)^{-n} D^{(p-1)(n-2 i)}: H^{n}\left(G, \mathbb{F}_{p}\right) \longrightarrow H^{n+2(p-1) i}\left(G, \mathbb{F}_{p}\right)
$$

with $m=\frac{p-1}{2}$ for $p$ odd.

We will have to use a more diagrammatic method to express the above in order to be able to prove that the Steenrod operations commute with the action of selfequivalences of the derived category.

As above, let $\mathbf{P}$ be a projective resolution of $R$ as $R G$-module and let $\mathbf{Q}$ be a projective resolution of $R$ as $R G \imath \mathfrak{S}_{p}$-module. As usual, there is a mapping, unique up to homotopy, $\mathbf{Q} \stackrel{\tau}{\longrightarrow} \bigotimes_{1 \leq i \leq p} \mathbf{P}$, lifting the identity on $R$. Then,

$$
\begin{aligned}
\operatorname{Hom}_{R\left(G^{p}\right)}\left(\bigotimes_{1 \leq i \leq p} \mathbf{P},\left(\bigotimes_{1 \leq i \leq p} M\right)[p \cdot n]\right) & \simeq \operatorname{Hom}_{R\left(G^{p}\right)}\left(\bigotimes_{1 \leq i \leq p} \mathbf{P}, \bigotimes_{1 \leq i \leq p} M[n]\right) \\
& \simeq \bigotimes_{1 \leq i \leq p} \operatorname{Hom}_{R G}(\mathbf{P}, M[n])
\end{aligned}
$$

with obvious isomorphisms. Observe that since the action of $\mathfrak{S}_{p}$ does not enter the picture yet, the sign representation does not play a rôle here.

With the diagonal $\Delta$ we get

$$
\operatorname{Hom}_{R G}(\mathbf{P}, M[n]) \stackrel{\Delta}{\longrightarrow} \bigotimes_{1 \leq i \leq p} \operatorname{Hom}_{R G}(\mathbf{P}, M[n]),
$$

and we observe that the image of $\Delta$ under the isomorphism

$$
\operatorname{Hom}_{R\left(G^{p}\right)}\left(\bigotimes_{1 \leq i \leq p} \mathbf{P}, \bigotimes_{1 \leq i \leq p} M[n]\right) \simeq \bigotimes_{1 \leq i \leq p} \operatorname{Hom}_{R G}(\mathbf{P}, M[n])
$$

is in $\operatorname{Hom}_{R\left(G i \mathfrak{S}_{p}\right)}\left(\bigotimes_{1 \leq i \leq p} \mathbf{P},\left(\bigotimes_{1 \leq i \leq p} M[n]\right) \otimes_{R} R_{s}^{(n)}\right)$. Now, the restriction of the sign representation of $\mathfrak{S}_{p}$ to $C_{p}$ always is the trivial representation, for $p=$ 2 , since then $1=-1$, and for $p$ an odd prime, since then any $p$-cycle has even parity. Therefore, $\Delta$ factors through $\operatorname{res}_{G^{p}}^{G i \mathfrak{S}_{p}}$ by a mapping $\Delta^{\prime}$. So, the norm map 
norm $_{G}^{G \times C_{p}}$ can be seen as left composition of the left vertical homomorphisms in the following commutative diagram:

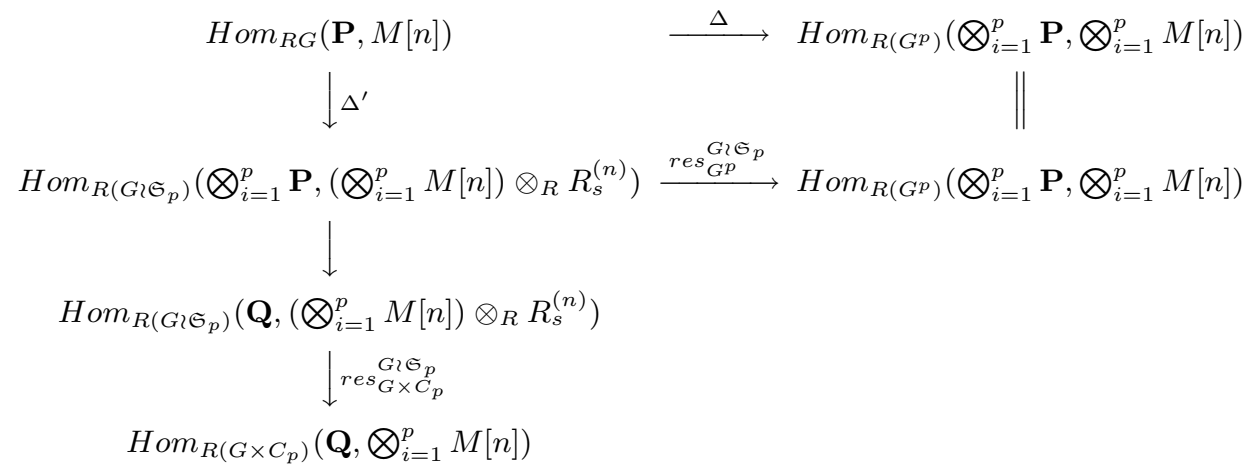

One should observe that $\Delta$ is not linear over the base ring unless this base ring $R$ is $\mathbb{F}_{p}$.

\section{Ring AUTOMORPHisms}

The group $\operatorname{Out}_{R}(R G)=\operatorname{Aut}_{R, 1}(R G) / \operatorname{Inn}(R G)$ of outer augmented $R$-algebra automorphisms acts on $H^{*}(G, R)$ in the obvious way. We shall prove that this action is compatible with Steenrod operations.

Proposition 2.1. Let $R=\mathbb{F}_{p}$. The action of $O u t_{\mathbb{F}_{p}}\left(\mathbb{F}_{p} G\right)$ on $H^{*}\left(G, \mathbb{F}_{p}\right)$ commutes with the action of mod $p$ Steenrod operations on $H^{*}\left(G, \mathbb{F}_{p}\right)$.

To prove this, we shall show that for any automorphism $\alpha$ of $\mathbb{F}_{p} G$ there is an automorphism $\hat{\alpha}$ of $\mathbb{F}_{p}\left(G<\mathfrak{S}_{p}\right)$ so that the diagram

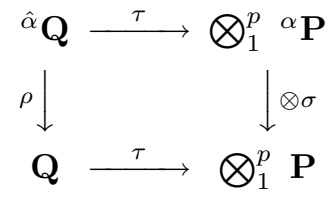

commutes. Here, the vertical homomorphisms are chain maps which lift the identity on $\mathbb{F}_{p}$; the complex $\mathbf{Q}$ is a projective resolution of $\mathbb{F}_{p}$, and a mapping $\rho$ lifting the identity on $\mathbb{F}_{p}$ exists by the universal property of $\mathbf{Q}$ being a projective resolution. $\mathbf{P}$ is a projective resolution of $\mathbb{F}_{p}$ as $G$-module. Then, we use the tensor product of $p$ copies of the lifting $\sigma$ of the identity on $\mathbb{F}_{p}$ onto $\mathbf{P}$ as a complex of $\mathbb{F}_{p} G$-modules. This tensor product is easily seen to be $\mathbb{F}_{p}\left(G<\mathfrak{S}_{p}\right)$-linear.

In order to define $\hat{\alpha}$ we observe the well known fact that

$$
\mathbb{F}_{p}\left(G \succ \mathfrak{S}_{p}\right) \simeq \mathbb{F}_{p} \underbrace{(G \times G \times \cdots \times G)}_{p \text { factors }} \otimes_{\mathbb{F}_{p}} \mathbb{F}_{p} \mathfrak{S}_{p}
$$

is isomorphic to the skew tensor product of the first factor with the quotient group acted upon: $\mathbb{F}_{p}\left(G<\mathfrak{S}_{p}\right)$ is the ordinary tensor product as an $\mathbb{F}_{p}$-module, and the multiplication is defined via a torsion by $\mathfrak{S}_{p}$ :

$$
\left(g_{1} \otimes \sigma_{1}\right) \cdot\left(g_{2} \otimes \sigma_{2}\right)=g_{1} \sigma_{1}\left(g_{2}\right) \otimes \sigma_{1} \sigma_{2} .
$$


Since $\mathbb{F}_{p} \underbrace{(G \times G \times \cdots \times G)}_{p \text { factors }} \simeq \bigotimes_{i=1}^{p} \mathbb{F}_{p} G$, we may take $\hat{\alpha}:=\left(\bigotimes_{i=1}^{p} \alpha\right) \otimes i d_{\mathfrak{S}_{p}}$, and we verify readily that $\hat{\alpha}$ is a ring automorphism of $\left.\mathbb{F}_{p}(G) \mathfrak{S}_{p}\right)$. Moreover, a projective resolution of $\bigotimes_{i=1}^{p} \mathbb{F}_{p}$ as an $\mathbb{F}_{p}\left(G \succ \mathfrak{S}_{p}\right)$-module is $\mathbf{Q}:=\left(\bigotimes_{i=1}^{p} \mathbf{P}\right) \otimes_{\mathbb{F}_{p}} \mathbf{R}$, where $\mathbf{P}$ is a projective resolution of $\mathbb{F}_{p}$ as an $\mathbb{F}_{p} G$-module and $\mathbf{R}$ is a projective resolution of $\mathbb{F}_{p}$ as an $\mathbb{F}_{p} \mathfrak{S}_{p}$-module (cf. e.g. [1] Chapter 4.1]). Note that since the Sylow $p$-subgroup of $\mathfrak{S}_{p}$ is cyclic, the homogeneous components of $\mathbf{R}$ are indecomposable projective $\mathbb{F}_{p} \mathfrak{S}_{p}$-modules.

We shall have to compare the diagram

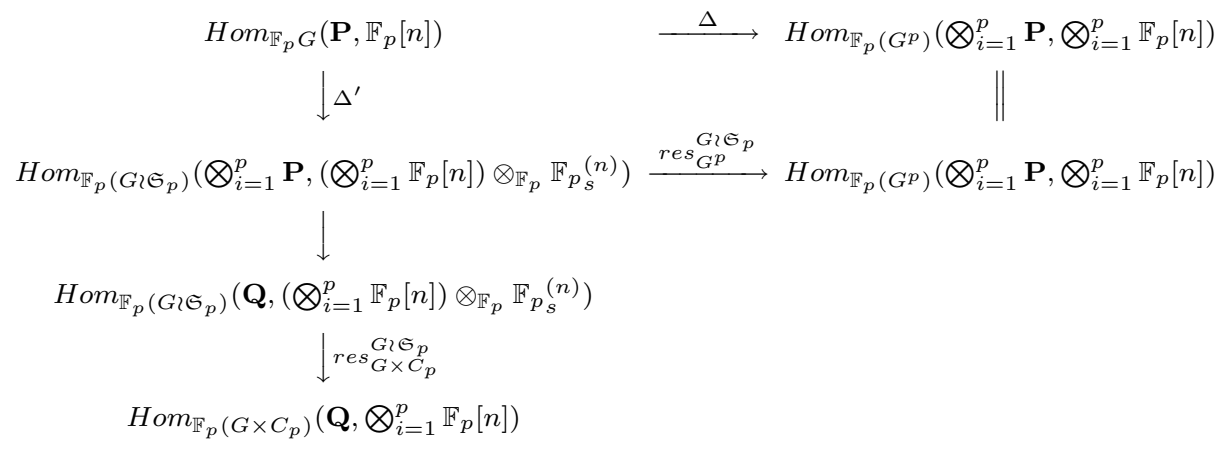

with the diagram corresponding to the twisted resolutions

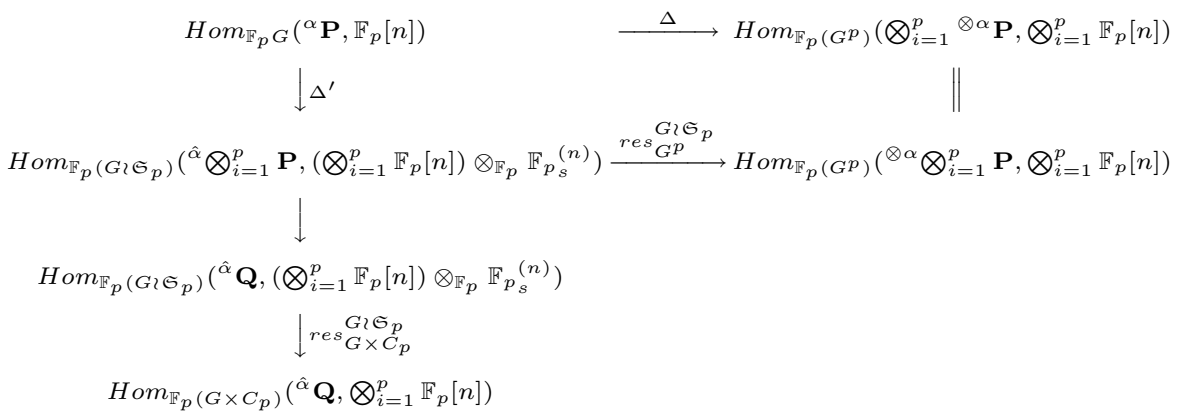

and we shall have to define mappings from the morphism groups of the bottom diagram to the corresponding morphism sets of the top diagram in such a way that the squares which are created this way are commutative. Since $\mathbf{P}$ as well as ${ }^{\alpha} \mathbf{P}$ are projective resolutions of the trivial module, there is a chain homotopy isomorphism $\sigma: \mathbf{P} \longrightarrow{ }^{\alpha} \mathbf{P}$ and this isomorphism induces a chain homotopy isomorphism $\bigotimes_{1}^{p} \sigma$ : $\bigotimes_{i=1}^{p} \mathbf{P} \longrightarrow \bigotimes_{i=1}^{p}{ }^{\alpha} \mathbf{P}$ and a chain homotopy isomorphism $\rho: \mathbf{Q} \longrightarrow{ }^{\hat{\alpha}} \mathbf{Q}$ defined by taking the identity on the factors corresponding to $\mathbf{R}$. It is then straightforward to see that all squares which occur are commutative.

In order to compute

$$
\operatorname{norm}_{G}^{G \times C_{p}}: H^{n}\left(G, \mathbb{F}_{p}\right) \longrightarrow H^{p n}\left(G \times C_{p}, \mathbb{F}_{p}\right)
$$

we have to map a cocycle by $\operatorname{res}_{G \times C_{p}}^{G i \mathfrak{S}_{p}} \circ \tau^{*} \circ \Delta^{\prime}$. 
Since $\operatorname{res}_{G \times C_{p}}^{G i \mathfrak{S}_{p}}=\delta^{*}$ for $\delta$ being the diagonal embedding of $G$ into $G^{p}$, and since $\hat{\alpha} \circ \delta=\delta \circ\left(\alpha \times i d_{C_{p}}\right)$,

$$
\begin{aligned}
\operatorname{norm}_{G}^{G \times C_{p}} \circ \alpha^{*} & =\operatorname{res}_{G \times C_{p}}^{G i \mathfrak{S}_{p}} \circ \tau^{*} \circ \Delta^{\prime} \circ \alpha^{*} \\
& =\operatorname{res}_{G \times C_{p}}^{G i \mathfrak{S}_{p}} \circ \hat{\alpha}^{*} \circ \tau^{*} \circ \Delta^{\prime} \\
& =\left(\alpha \times i d_{C_{p}}\right)^{*} \circ \operatorname{res}_{G \times C_{p}}^{G i \mathfrak{S}_{p}} \circ \tau^{*} \circ \Delta^{\prime} \\
& =\left(\alpha \times i d_{C_{p}}\right)^{*} \circ \operatorname{norm}_{G}^{G \times C_{p}} .
\end{aligned}
$$

The proof is complete.

\section{DeRIVED SELF-EQUiVALENCES}

Let $R$ be a field of characteristic $p$, let $B(R G)$ be the principal block of $R G$ and let $X$ be a two-sided tilting complex in $D^{b}\left(B(R G) \otimes_{R} B(R G)^{o p}\right)$. Starting from $X$, we shall construct a two-sided tilting complex in $D^{b}\left(B\left(R G \succ \mathfrak{S}_{p}\right) \otimes_{R} B\left(R G \imath \mathfrak{S}_{p}\right)^{o p}\right)$.

It should be noted that Andrei Marcus constructed in [6] a tilting complex in $B\left(R G \succ \mathfrak{S}_{n}\right)$ if $p$ does not divide $n$. However, in order to apply this construction to the Steenrod operation we are obliged to pass to $n=p$.

We consider the complex

$$
X^{\otimes p}:=X \otimes_{R} X \otimes_{R} \cdots \otimes_{R} X
$$

with $p$ factors as an object in $D^{b}\left(B\left(R G^{p}\right) \otimes_{R} B\left(R G^{p}\right)^{o p}\right)$. Of course, this is an invertible complex. As for the endomorphism complex, we get

$$
\operatorname{Hom}_{R G^{p}}\left(X^{\otimes p}, X^{\otimes p}\right) \simeq \bigotimes_{i=1}^{p} \operatorname{Hom}_{R G}(X, X) \simeq \bigotimes_{i=1}^{p} B(R G)
$$

in $D^{b}\left(R G^{p} \otimes_{R}\left(R G^{p}\right)^{o p}\right)$.

We consider now the complex of $R\left(G<\mathfrak{S}_{p}\right)$-right modules

$$
X^{G \imath \mathfrak{S}_{p}}:=X^{\otimes p} \otimes_{R G^{p}} R\left(G \prec \mathfrak{S}_{p}\right) .
$$

It is immediate that $X^{G \imath \mathfrak{S}_{p}} \simeq X^{\otimes p} \otimes_{R} R \mathfrak{S}_{p}$ as $R\left(G^{p}\right)$-left modules, where the $p$ factors of $G \times G \times \ldots \cdots \times G$ act on the respective copies of $X \otimes X \otimes \cdots \otimes X$ respectively and each acts trivially on $R \mathfrak{S}_{p}$.

We shall now define a left action of $R\left(G<\mathfrak{S}_{p}\right)$ on $X^{G \imath \mathfrak{S}_{p}}$ which commutes with the right action of $G<\mathfrak{S}_{p}$ (see [1, Chapter 4.1]).

Let $\sigma$ be an element of $\mathfrak{S}_{p}$. The degree $n$ homogeneous component of $X^{\otimes p}$ is

$$
\left(X^{\otimes p}\right)_{n}=\bigoplus_{k_{1}+\cdots+k_{p}=n} X_{k_{1}} \otimes_{R} X_{k_{2}} \otimes_{R} \cdots \otimes_{R} X_{k_{p}} .
$$

The differential $\delta_{n}$ on the tensor product complex is

$$
\begin{aligned}
& \sum_{k_{1}+\cdots+k_{p}=n} d \otimes i d \otimes \cdots \otimes i d+(-1)^{k_{1}} i d \otimes d_{n-k} \otimes i d \otimes \cdots \otimes i d \\
& +\cdots+(-1)^{k_{1}+\cdots+k_{p-1}} i d \otimes \cdots \otimes i d \otimes d .
\end{aligned}
$$

Now, $\sigma$ acts on the tensor product complex permuting the factors

$$
x_{1} \otimes \cdots \otimes x_{p} \in X_{k_{1}} \otimes_{R} X_{k_{2}} \otimes_{R} \cdots \otimes_{R} X_{k_{p}}
$$


accordingly as $\sigma$ acts on the index set $\{1, \ldots, p\}$ multiplied by a sign $(-1)^{\nu}$, where

$$
\nu=\sum_{j<k ; \sigma(j)>\sigma(k)} \operatorname{deg}\left(x_{j}\right) \operatorname{deg}\left(x_{k}\right) .
$$

A more standard definition of $X^{G i \mathfrak{S}_{p}}$, by tensor induction followed by ordinary induction, can be done using the fact that $X$ itself is a complex of $G \times G$-modules.

Let $H$ be a finite group and let $Y$ be a complex of $R H$-modules. Then, the tensor induced complex $Y^{\otimes \uparrow_{H}^{H \times C_{p}}}$ is isomorphic to $\underbrace{Y \otimes_{R} Y \otimes_{R} \cdots \otimes_{R} Y}_{p \text { factors }}$ as a complex of $R\left(H \times C_{p}\right)$-modules. But actually, $Y^{\otimes \uparrow_{H}^{H \times C_{p}}}$ is a complex of $R\left(H \prec \mathfrak{S}_{p}\right)$-modules, as is seen easily from the definition (cf. e.g. [1]).

We will not use the two following lemmas, though we cite them since they might become important in later studies.

Lemma 3.1. Let $S$ be a p-subgroup of $H$. If $Y$ is a direct sum of relatively $S$ projective $H$-modules, then $Y^{\otimes \uparrow_{H}^{H \times C_{p}}}$ is relatively $S$ 々 $C_{p}$-projective.

Proof. Let $U$ be an $R S$-module, so that $Y \mid U \uparrow_{S}^{H}$. Let $\pi: U \uparrow_{S}^{H} \longrightarrow Y$ and $\iota: Y \longrightarrow U \uparrow_{S}^{H}$ with $\pi \circ \iota=i d_{Y}$. Then, the monomorphism

$$
\underbrace{Y \otimes_{R} Y \otimes_{R} \cdots \otimes_{R} Y}_{p \text { factors }} \stackrel{\iota \otimes \cdots \otimes_{\iota}}{\longrightarrow} \underbrace{U \uparrow_{S}^{H} \otimes_{R} U \uparrow_{S}^{H} \otimes_{R} \cdots \otimes_{R} U \uparrow_{S}^{H}}_{p \text { factors }}
$$

is split by $\pi \otimes \pi \otimes \cdots \otimes \pi$, and both morphisms are clearly morphisms of $R\left(H \curlywedge \mathfrak{S}_{p}\right)$ modules.

Moreover,

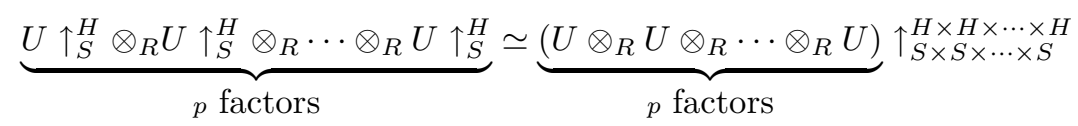

as $R(H \times H \times \cdots \times H)$-modules. Nevertheless, the isomorphism consists of reidentifying the order of the tensors, and this isomorphism is clearly $H$ ح $\mathfrak{S}_{p}$-linear. But now, the isomorphism induces an isomorphism

$$
\underbrace{U \uparrow_{S}^{H} \otimes_{R} U \uparrow_{S}^{H} \otimes_{R} \cdots \otimes_{R} U \uparrow_{S}^{H}}_{p \text { factors }} \simeq \underbrace{\left(U \otimes_{R} U \otimes_{R} \cdots \otimes_{R} U\right)}_{p \text { factors }} \uparrow_{S i \mathfrak{S}_{p}}^{H i \mathfrak{S}_{p}} .
$$

Since $Y$ is a direct factor of this induced module, and since $C_{p}$ is the Sylow $p$ subgroup of $\mathfrak{S}_{p}$, the result follows.

We observe that

$$
X^{G \imath \mathfrak{S}_{p}}:=\left(X^{\otimes \uparrow_{G \times G}^{(G \times G) \times C_{p}}}\right) \uparrow_{(G \times G) \imath \mathfrak{S}_{p}}^{\left(G \imath \mathfrak{S}_{p}\right) \times\left(G \imath \mathfrak{S}_{p}\right)}
$$

Indeed, $\left(X^{\otimes \uparrow_{G \times G}^{(G \times G) \times C_{p}}}\right)$ is a tensor product of $p$ copies of $X$, the direct product of $p$ copies of $G$ acting from the left and from the right on the different copies of the $p$-fold tensor product, and on which $C_{p}$ acts from the left and from the right by permuting the factors of the tensor product. Moreover, $(G \times G) \imath \mathfrak{S}_{p}$ embeds into $\left(G \succ \mathfrak{S}_{p}\right) \times\left(G \succ \mathfrak{S}_{p}\right)$ via the mapping $\left(g_{1}, g_{2}, c\right) \mapsto\left(g_{1}, c\right) \times\left(g_{2}, c\right)$, where $g_{1}, g_{2} \in G^{p}$. It is immediate that this construction gives the one defined above in a more elementary manner. However, we have not looked at the differentials yet. Here, either we deal 
with multicomplexes and take the total complex of these multicomplexes, or we have to go back to the elementary definition above.

Lemma 3.2. Let $P$ be a Sylow p-subgroup of $G$. If $X$ is a direct sum of $\Delta(P)$ projective modules, then $X^{G \imath \mathfrak{S}_{p}}$ is a direct sum of $\Delta\left(P \backslash C_{p}\right)$-projective modules.

Proof. Let $P$ be a $p$-Sylow subgroup of $G$. Then, $P^{p}$ is a $p$-Sylow subgroup of $G^{p}$ and $P \imath C_{p}$ is a $p$-Sylow subgroup of $G \imath \mathfrak{S}_{p}$. Let $\Delta(P)$ be the diagonal of $P$ in $G \times G$. Since by Lemma $3.1\left(X^{\otimes \uparrow_{G \times G}^{(G \times G) \times C_{p}}}\right)$ is $\Delta\left(P^{p}\right) \rtimes C_{p}$-projective, $X^{G \imath \mathfrak{S}_{p}}$ is $\Delta\left(P \imath C_{p}\right)$ projective. Indeed, $C_{p}$ in $\Delta\left(P^{p}\right) \rtimes C_{p}$ is diagonally embedded in $\left(G \imath \mathfrak{S}_{p}\right) \times\left(G \imath \mathfrak{S}_{p}\right)$ and $X^{G \imath \mathfrak{S}_{p}}$ is induced from $(G \times G)^{p} \rtimes C_{p}$. So, if $\left(X^{\otimes \uparrow_{G \times G}^{(G \times G) \times C_{p}}}\right)$ is $\Delta\left(P^{p}\right) \rtimes C_{p^{-}}$ projective, $X^{G \imath \mathfrak{S}_{p}}$ is $\Delta\left(P \nmid C_{p}\right)$-projective.

The result follows from this.

Proposition 3.3. Let $R$ be a field of characteristic $p$, and $G$ a finite group with Sylow $p$ subgroup $P$. Then, for any $X$ with isomorphism class in $\operatorname{TrPic} R(B(R G))$, the complex $X^{G i \mathfrak{S}_{p}}$ is a two-sided tilting complex in $\operatorname{TrPic} R\left(B\left(R\left(G \succ \mathfrak{S}_{p}\right)\right)\right)$.

If $X$ is splendid, then $X^{G i \mathfrak{S}_{p}}$ is a two-sided tilting complex with homogeneous components which are $\Delta(P)$-projective p-permutation modules.

Remark 3.4. The complex $X^{G \imath \mathfrak{S}_{p}}$ is in general not splendid even if $X$ is splendid. This surprising fact can be seen if one wants to apply Brauer functors with respect to the Sylow $p$-subgroup of $\mathfrak{S}_{p}$. The complex $X^{G / \mathfrak{S}_{p}}$ will then be transformed into a complex which is not a tilting complex anymore.

Proof of Proposition 3.3. We will show that the above defined complex $X^{G i \mathfrak{S}_{p}}$ is a two-sided tilting complex in $D^{b}\left(B\left(R\left(G \succ \mathfrak{S}_{p}\right)\right) \otimes_{R} B\left(R\left(G \succ \mathfrak{S}_{p}\right)\right)^{o p}\right)$.

We get isomorphisms of (the total complexes of) double complexes

$$
\begin{aligned}
\operatorname{Hom}_{R\left(G \backslash \mathfrak{S}_{p}\right)}^{\bullet}\left(X^{G i \mathfrak{S}_{p}}, X^{G \imath \mathfrak{S}_{p}}\right) & \simeq \operatorname{Hom}_{R\left(G^{p}\right)}^{\bullet}\left(X^{\otimes p}, X^{G i \mathfrak{S}_{p}} \downarrow_{G^{p}}\right) \\
& \simeq \bigoplus_{\sigma \in \mathfrak{S}_{p}} \operatorname{Hom}_{R\left(G^{p}\right)}^{\bullet}\left(X^{\otimes p}, X^{\otimes p}\right) \otimes \sigma \\
& \simeq \bigoplus_{\sigma \in \mathfrak{S}_{p}}\left(\bigotimes_{i=1}^{p} \operatorname{Hom}_{R G}^{\bullet}(X, X)\right) \otimes \sigma
\end{aligned}
$$

Moreover, we have to recover the action of $R\left(G \succ \mathfrak{S}_{p}\right)$ coming from the left action of $R\left(G<\mathfrak{S}_{p}\right)$ on the first and on the second variable $X^{G / \mathfrak{S}_{p}}$. The action on the first variable is going to become the right action of $R\left(G \prec \mathfrak{S}_{p}\right)$ on $\operatorname{Hom}_{R\left(G \imath \mathfrak{S}_{p}\right)}^{\bullet}\left(X^{G \imath \mathfrak{S}_{p}}, X^{G i \mathfrak{S}_{p}}\right)$, and the action on the second variable is going to become the left action on this Hom-complex.

The action of the subgroup $G^{p}$ of $G \succ \mathfrak{S}_{p}$ trivially is factorwise on the tensor products, on each of the direct summands. The action of $\mathfrak{S}_{p}$ from the right on the complex $X^{G l \mathfrak{S}_{p}}$ is given by the induction

$$
X^{\otimes p} \uparrow_{G^{2} \imath \mathfrak{S}_{p}}^{\left(G i \mathfrak{S}_{p}\right)^{2}} \simeq X^{\otimes p} \otimes_{R} R \mathfrak{S}_{p}
$$

The action from the right on the $\mathrm{Hom}^{\bullet}$-complex comes from the action on the second variable, which is, after applying Frobenius reciprocity, just the above. Hence 
the action of $\mathfrak{S}_{p}$ from the right permutes the direct summands of

$$
\bigoplus_{\sigma \in \mathfrak{S}_{p}}\left(\bigotimes_{i=1}^{p} H o m_{R G}^{\bullet}(X, X)\right) \otimes \sigma
$$

We shall study the left action of $G \prec \mathfrak{S}_{p}$ on the Hom-complex. The action of $G^{p} \leq G<\mathfrak{S}_{p}$ on the left trivially is again the factorwise action on the two factors of the tensor products, in each of the direct summands. Now, the action of $\sigma \in \mathfrak{S}_{p}$ from the left permutes the factors in the tensor product on the level of the modules in each degree, and applies a sign according to the above formula, and henceforth also the factors in the tensor product

$$
\bigoplus_{\sigma \in \mathfrak{S}_{p}}\left(\bigotimes_{i=1}^{p} H o m_{R G}^{\bullet}(X, X)\right) \otimes \sigma
$$

Now, we may assume the homogeneous components of $X$ are free as $B(R G) \otimes_{R}$ $B(R G)^{o p}$-modules, except the leftmost one, which is projective as an $R G$-right module and projective as an $R G$-left module. Then, using the Künneth formula,

$$
H^{0}\left(\operatorname{Hom}_{R\left(G \imath \mathfrak{S}_{p}\right)}^{\bullet}\left(X^{G \imath \mathfrak{S}_{p}}, X^{G \imath \mathfrak{S}_{p}}\right)\right)=\left(\bigotimes_{i=1}^{p} \operatorname{Hom}_{K(R G)}(X, X)\right) \otimes_{R} R \mathfrak{S}_{p},
$$

and $H^{n}\left(\operatorname{Hom}_{R G}^{\bullet}\left(X^{G i \mathfrak{S}_{p}}, X^{G l \mathfrak{S}_{p}}\right)\right)=0$ for $n \neq 0$, again by the Künneth formula. The Künneth formula applies without problems since $R$ is a field. Then, at the final step we use that $X$ is a tilting complex from either side. So, $\operatorname{Hom}_{R G}^{\bullet}\left(X^{G i \mathfrak{S}_{p}}, X^{G i \mathfrak{S}_{p}}\right)$ is quasi-isomorphic to its degree 0 homology as left module and as right module. Therefore, by ordinary Morita theory (see e.g. Curtis-Reiner [2, (3.54)]) $\operatorname{Hom}_{R\left(G \imath \mathfrak{S}_{p}\right)}\left(X^{G \imath \mathfrak{S}_{p}}, X^{\left.G \imath \mathfrak{S}_{p}\right)}\right.$ is an invertible $B\left(R\left(G \imath \mathfrak{S}_{p}\right)\right)$-bimodule. This implies that $X^{G \imath \mathfrak{S}_{p}}$ is a two-sided tilting complex in $\operatorname{Tr} P i c_{R}\left(B\left(R\left(G \imath \mathfrak{S}_{p}\right)\right)\right)$. As a consequence, since $R\left(G \imath \mathfrak{S}_{p}\right)$ is a symmetric algebra,

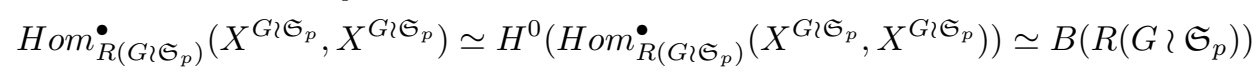
in the derived category of $R\left(G \imath \mathfrak{S}_{p}\right) \otimes_{R} R\left(G \imath \mathfrak{S}_{p}\right)^{o p}$-modules.

By Lemma 3.2, if $X$ is a complex of $p$-permutation modules over $G \times G$ which are induced from the diagonal of a fixed $p$-Sylow subgroup of $G$, then $X^{G i \mathfrak{S}_{p}}$ also is a complex of $p$-permutation modules over $\left(G \imath \mathfrak{S}_{p}\right) \times\left(G \imath \mathfrak{S}_{p}\right)$, induced from a fixed Sylow $p$-subgroup of $G \imath \mathfrak{S}_{p}$.

Proposition 3.3 follows.

Remark 3.5. It is straightforward to generalize the statement and the proof of Proposition 3.3 to the case where $X$ is a two-sided tilting complex between two blocks $B$ and $b$ of $R G$, and $R H$ for two finite groups $G$ and $H$, and $R$ is a field of finite characteristic. Moreover, it is an easy task to pass to any wreath product by a fixed permutation group $\Pi$.

We now study first properties of $X^{G \imath \mathfrak{S}_{p}}$ with respect to the action on Extalgebras [11.

Lemma 3.6. Let $M$ be an $R G$-module for a finite group $G$ and let $X$ be a tilting complex with isomorphism class in $H D_{M}(G)$. Let $R_{s}$ be the sign representation of $\mathfrak{S}_{p}$. Then, the isomorphism class of $X^{G i \mathfrak{S}_{p}}$ is in $H D_{\left(\otimes_{i=1}^{p} M\right)}\left(G \succ \mathfrak{S}_{p}\right) \cap$ $H D_{\left(\left(\otimes_{i=1}^{p} M\right) \otimes_{R} R_{s}\right)}\left(G \succ \mathfrak{S}_{p}\right)$. 
Proof. We have

$$
\begin{aligned}
X^{G i \mathfrak{S}_{p}} \otimes_{G i \mathfrak{S}_{p}}\left(\bigotimes_{i=1}^{p} M\right) & \simeq\left(X^{\otimes p} \otimes_{R} R \mathfrak{S}_{p}\right) \otimes_{G^{p} \rtimes \mathfrak{S}_{p}}\left(\bigotimes_{i=1}^{p} M\right) \\
& \simeq\left(X^{\otimes p}\right) \otimes_{G^{p}}\left(\bigotimes_{i=1}^{p} M\right) \\
& \simeq \bigotimes_{i=1}^{p}\left(X \otimes_{G} M\right) \\
& \simeq \bigotimes_{i=1}^{p} M,
\end{aligned}
$$

where the last is an isomorphism in the derived category. Moreover,

$$
\begin{aligned}
X^{G i \mathfrak{S}_{p}} \otimes_{G i \mathfrak{S}_{p}}\left(\left(\bigotimes_{i=1}^{p} M\right) \otimes_{R} R_{s}\right) & \simeq\left(X^{\otimes p} \otimes_{R} R \mathfrak{S}_{p}\right) \otimes_{G^{p} \rtimes \mathfrak{S}_{p}}\left(\left(\bigotimes_{i=1}^{p} M\right) \otimes_{R} R_{s}\right) \\
& \simeq\left(\left(X^{\otimes p}\right) \otimes_{G^{p}}\left(\bigotimes_{i=1}^{p} M\right)\right) \otimes_{R}\left(R \mathfrak{S}_{p} \otimes_{\mathfrak{S}_{p}} R_{s}\right) \\
& \simeq\left(\bigotimes_{i=1}^{p}\left(X \otimes_{G} M\right)\right) \otimes_{R} R_{s} \\
& \simeq\left(\bigotimes_{i=1}^{p} M\right) \otimes_{R} R_{s}
\end{aligned}
$$

is a similar computation. This proves the statement.

We are now able to prove that the mod 2 Steenrod square $S q$ and the $\bmod p$ Steenrod operation $P$ commute with the action of $H D_{R}(G)$.

Theorem 1. Let $G$ be a finite group, and let $R$ be a field of characteristic $p$. Then, for any $X$ with isomorphism class in $H D_{R}(G)$, the following squares are commutative. If $p=2$, then

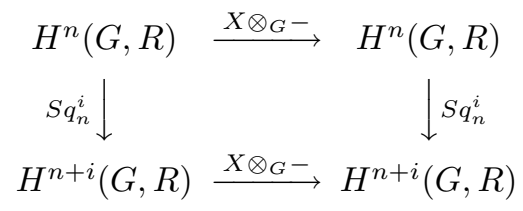

is commutative, and if $p \geq 3$, then

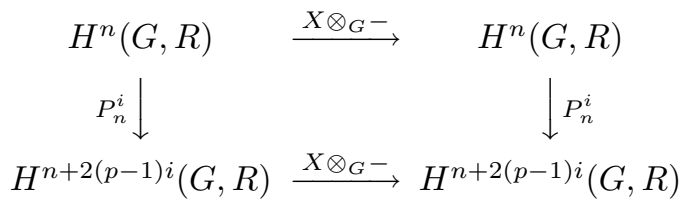

is commutative.

Proof. We use the interpretation of the Steenrod operation from section 1.2 We have to apply $X \otimes_{G}-$ or $X^{G i \mathfrak{S}_{p}} \otimes_{G \imath \mathfrak{S}_{p}}-$ or $\left(X \otimes_{R} R C_{p}\right) \otimes_{G \times C_{p}}$ to the various 
mappings in the diagram

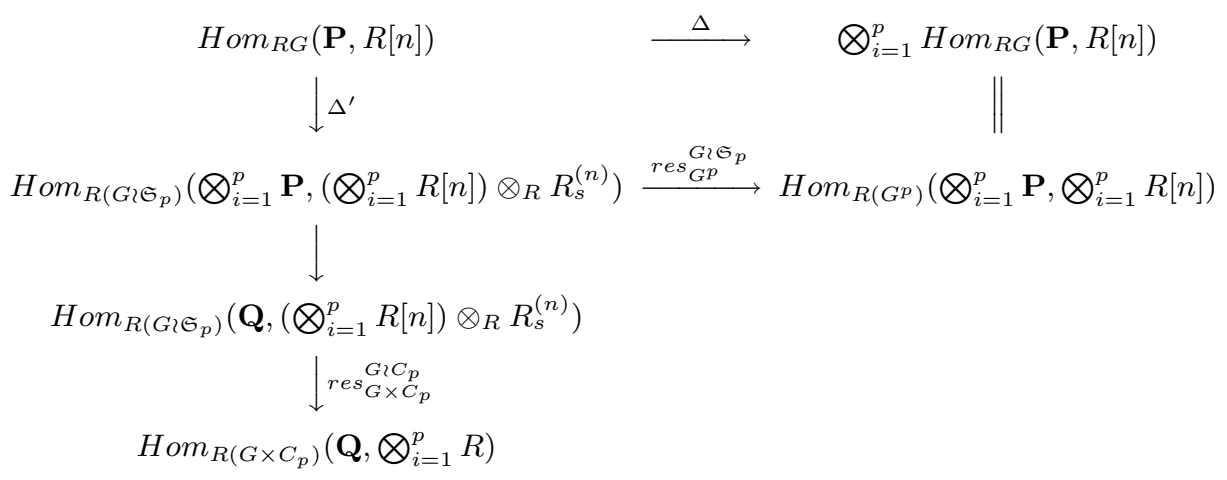

and shall prove that the squares which are induced by this are commutative in each case.

The square

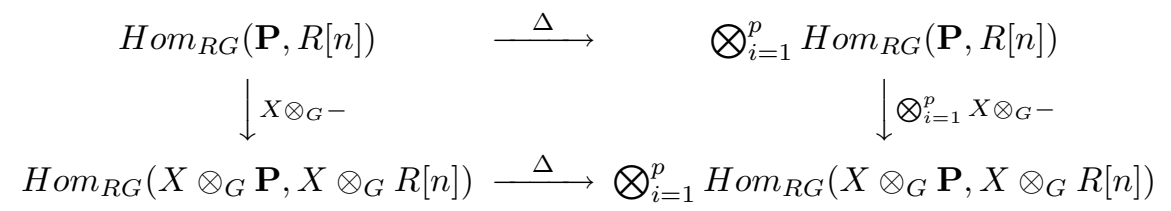

is obviously commutative. Moreover, the square

$$
\begin{array}{ccc}
\bigotimes_{i=1}^{p} \operatorname{Hom}_{R G}(\mathbf{P}, R[n]) & \simeq \operatorname{Hom}_{R G^{p}}\left(\bigotimes_{i=1}^{p} \mathbf{P}, \bigotimes_{i=1}^{p} R[n]\right) \\
\bigotimes_{i=1}^{p}\left(X \otimes_{G}-\right) \downarrow & \downarrow X^{\otimes p} \otimes_{G^{p}}- \\
\bigotimes_{i=1}^{p} \operatorname{Hom}_{R G}\left(X \otimes_{G} \mathbf{P}, X \otimes_{G} R[n]\right) & \simeq \operatorname{Hom}_{R G^{p}}\left(\bigotimes_{i=1}^{p} \mathbf{P}, \bigotimes_{i=1}^{p} R[n]\right)
\end{array}
$$

is commutative as well. This proves the announced commutativity of the diagrams with respect to the mappings $\Delta$ and $\Delta^{\prime}$.

The square

$$
\begin{aligned}
& \operatorname{Hom}_{R\left(G i \mathfrak{S}_{p}\right)}\left(\bigotimes_{i=1}^{p} \mathbf{P}, \bigotimes_{i=1}^{p} R[n] \otimes_{R} R_{s}^{(n)}\right) \quad \stackrel{\operatorname{res}_{G p}^{G i C_{p}}}{\longrightarrow} \operatorname{Hom}_{R\left(G^{p}\right)}\left(\bigotimes_{i=1}^{p} \mathbf{P}, \bigotimes_{i=1}^{p} R[n]\right) \\
& \downarrow X^{G i \mathfrak{S}_{p}} \otimes_{G \mathfrak{G} \mathfrak{S}_{p}-} \quad \downarrow X^{\otimes p} \otimes_{G^{p}}-
\end{aligned}
$$

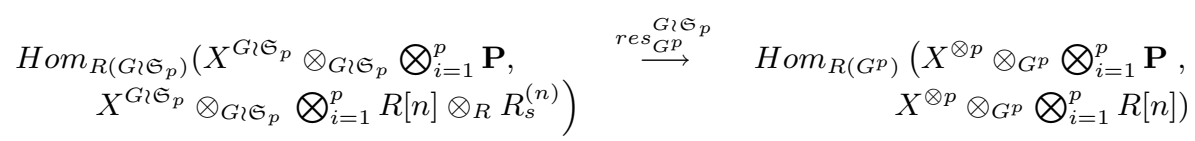

again is commutative. In fact the complex $X^{G \imath \mathfrak{S}_{p}}$ is an induced complex. And so, $X^{G \imath \mathfrak{S}_{p}} \otimes_{G l \mathfrak{S}_{p}}-$ is the same as $X^{\otimes p} \otimes_{G^{p}}-$. This proves that applying the various two-sided tilting complexes derived from $X$ to the upper square of the original diagram yields a parallel square which, together with the mappings of the corresponding corners, forms a commutative cube. 
Next, the square

$$
\begin{array}{ccc}
\operatorname{Hom}_{R\left(G \imath \mathfrak{S}_{p}\right)}\left(\mathbf{Q}, \otimes_{i=1}^{p} R[n] \otimes_{R} R_{s}^{(n)}\right) & \longrightarrow & \operatorname{Hom}_{R\left(G^{p}\right)}\left(\otimes_{i=1}^{p} \mathbf{P}, \otimes_{i=1}^{p} R[n]\right) \\
\downarrow X^{G \imath \mathfrak{S}_{p}} \otimes_{G i \mathfrak{S}_{p}}- & & \downarrow X^{\otimes p} \otimes_{G^{p}}- \\
\operatorname{Hom}_{R\left(G \imath \mathfrak{S}_{p}\right)}\left(X^{G \imath \mathfrak{S}_{p}} \otimes_{G \imath \mathfrak{S}_{p}} \mathbf{Q},\right. & \longrightarrow & \operatorname{Hom}_{R\left(G^{p}\right)}\left(X^{\otimes p} \otimes_{G^{p}} \otimes_{i=1}^{p} \mathbf{P},\right. \\
\left.X^{G \imath \mathfrak{S}_{p}} \otimes_{G \imath \mathfrak{S}_{p}} \otimes_{i=1}^{p} R[n] \otimes_{R} R_{s}^{(n)}\right) & & \left.X p \otimes_{G^{p}} \otimes_{i=1}^{p} R[n]\right)
\end{array}
$$

is commutative, for the same trivial reasons.

The only part which remains to be proven is that the square

$(*)$

$$
\begin{aligned}
& \operatorname{Hom}_{R\left(G\left\{\mathfrak{S}_{p}\right)\right.}\left(\mathbf{Q}, \bigotimes_{i=1}^{p} R[n] \otimes_{R} R_{s}^{(n)}\right) \quad \stackrel{\substack{G i \mathfrak{S}_{p} \\
\operatorname{res} \times C_{p}}}{\longrightarrow} \quad \operatorname{Hom}_{R\left(G \times \mathfrak{S}_{p}\right)}\left(\mathbf{Q}, \bigotimes_{i=1}^{p} R[n]\right) \\
& \downarrow X^{G \imath C_{p}} \otimes_{G \imath C_{p}}-\quad\left(X \otimes_{R} R C_{p}\right) \otimes_{G \times C_{p}}-\downarrow
\end{aligned}
$$

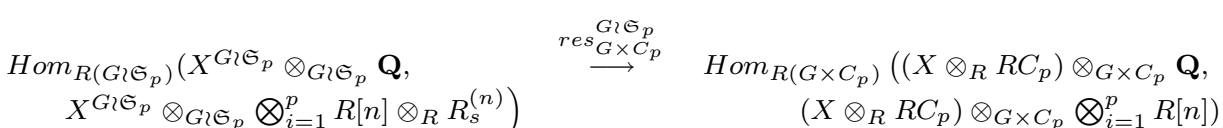

is commutative.

In order to prove this fact we replace $R$ by a fixed projective resolution. Let $\mathbf{P}$ be a projective resolution of $R$ as an $R G$-module and let $\mathbf{Q}$ be a minimal projective resolution of $R$ as an $R \mathfrak{S}_{p}$-module. Since $R \mathfrak{S}_{p}$ has cyclic defect group, each homogeneous component of $\mathbf{Q}$ is indecomposable. A projective resolution $\mathbf{P}^{G \imath \mathfrak{S}_{p}}$ of $R$ as an $R\left(G<\mathfrak{S}_{p}\right)$-module is then $\bigotimes_{i=1}^{p} \mathbf{P} \otimes_{R} \mathbf{Q}$, and a projective resolution of $R_{s}^{(n)}$ as an $R\left(G \succ \mathfrak{S}_{p}\right)$-module is $\bigotimes_{i=1}^{p} \mathbf{P} \otimes_{R}\left(\mathbf{Q} \otimes_{R} R_{s}^{(n)}\right)$. Note that $\mathbf{Q}_{s}:=\mathbf{Q} \otimes_{R} R_{s}^{(n)}$. We shall replace $R$ by this resolution $\mathbf{P}^{G \imath \mathfrak{S}_{p}}$ and $R_{s}^{(n)}$ by the resolution $\mathbf{P}^{G \imath \mathfrak{S}_{p}} \otimes_{R} R_{s}^{(n)}$.

Now,

$$
X^{G \imath \mathfrak{S}_{p}} \otimes_{G \imath \mathfrak{S}_{p}} \mathbf{P}^{G \imath \mathfrak{S}_{p}} \simeq X^{G \imath \mathfrak{S}_{p}} \otimes_{G \imath \mathfrak{S}_{p}}\left(\bigotimes_{i=1}^{p} \mathbf{P} \otimes_{R} \mathbf{Q}\right) \simeq\left(\bigotimes_{i=1}^{p}\left(X \otimes_{G} \mathbf{P}\right)\right) \otimes_{R} \mathbf{Q}
$$

and so, using Künneth formulas,

$$
\begin{aligned}
& \operatorname{Hom}_{K\left(R G \imath \mathfrak{S}_{p}\right)}\left(X^{G \imath \mathfrak{S}_{p}} \otimes_{G \imath \mathfrak{S}_{p}} \mathbf{P}^{G \imath \mathfrak{S}_{p}}, X^{G \imath \mathfrak{S}_{p}} \otimes_{G \imath \mathfrak{S}_{p}}\left(\mathbf{P}^{G \imath \mathfrak{S}_{p}} \otimes_{R} R_{s}^{(n)}\right)[n]\right) \\
& \simeq \operatorname{Hom}_{K\left(R G \imath \mathfrak{S}_{p}\right)}\left(\left(\bigotimes_{i=1}^{p}\left(X \otimes_{G} \mathbf{P}\right)\right) \otimes_{R} \mathbf{Q},\left(\bigotimes_{i=1}^{p}\left(X \otimes_{G} \mathbf{P}\right)\right) \otimes_{R} \mathbf{Q}_{s}[n]\right) \\
& \simeq \bigoplus_{i+j=n} H \operatorname{om}_{K\left(R G^{p}\right)}\left(\left(\bigotimes_{i=1}^{p}\left(X \otimes_{G} \mathbf{P}\right)\right),\left(\bigotimes_{i=1}^{p}\left(X \otimes_{G} \mathbf{P}\right)\right)[i]\right) \\
& \otimes_{R} \operatorname{Hom}_{K\left(R \mathfrak{S}_{p}\right)}\left(\mathbf{Q}, \mathbf{Q}_{s}[j]\right) \\
& \simeq \bigoplus_{i+j=n}\left(\bigotimes_{\sum_{l=1}^{p} i_{l}=i} \operatorname{Hom}_{K(R G)}\left(X \otimes_{G} \mathbf{P}, X \otimes_{G} \mathbf{P}\left[i_{l}\right]\right)\right) \\
& \otimes_{R} \operatorname{Hom}_{K\left(R \mathfrak{S}_{p}\right)}\left(\mathbf{Q}, \mathbf{Q}_{s}[j]\right)
\end{aligned}
$$


Similarly,

$$
\begin{aligned}
& \operatorname{Hom}_{K\left(R G \imath \mathfrak{S}_{p}\right)}\left(\mathbf{P}^{G i \mathfrak{S}_{p}}, \mathbf{P}^{G i \mathfrak{S}_{p}} \otimes_{R} R_{s}^{(n)}[n]\right) \\
& \simeq \operatorname{Hom}_{K\left(R G \imath \mathfrak{S}_{p}\right)}\left(\left(\bigotimes_{i=1}^{p} \mathbf{P}\right) \otimes_{R} \mathbf{Q},\left(\bigotimes_{i=1}^{p} \mathbf{P}\right) \otimes_{R} \mathbf{Q}_{s}[n]\right) \\
& \simeq \bigoplus_{i+j=n} \operatorname{Hom}_{K\left(R G^{p}\right)}\left(\bigotimes_{i=1}^{p} \mathbf{P},\left(\bigotimes_{i=1}^{p} \mathbf{P}\right)[i]\right) \otimes_{R} \operatorname{Hom}_{K\left(R \mathfrak{S}_{p}\right)}\left(\mathbf{Q}, \mathbf{Q}_{s}[j]\right) \\
& \simeq \bigoplus_{i+j=n}\left(\underset{\sum_{l=1}^{p} i_{l}=i}{\bigotimes_{K o m}(R G)}\left(\mathbf{P}, \mathbf{P}\left[i_{l}\right]\right)\right) \otimes_{R} \operatorname{Hom}_{K\left(R \mathfrak{S}_{p}\right)}\left(\mathbf{Q}, \mathbf{Q}_{s}[j]\right),
\end{aligned}
$$

with the same isomorphisms.

Now, we shall have to study how the restriction $\operatorname{res}_{G \times C_{p}}^{G i \mathfrak{S}_{p}}$ is interpreted in this context. Actually,

$$
\begin{aligned}
& r e s_{G \times C_{p}}^{G i \mathfrak{S}_{p}}\left(\operatorname{Hom}_{K\left(R G l \mathfrak{S}_{p}\right)}\left(\mathbf{P}^{G l \mathfrak{S}_{p}}, \mathbf{P}^{G l \mathfrak{S}_{p}} \otimes_{R} R_{s}^{(n)}[n]\right)\right) \\
& \quad \simeq \operatorname{res}_{G \times C_{p}}^{G i \mathfrak{S}_{p}}\left(\bigoplus_{i+j=n}\left(\bigotimes_{\sum_{l=1}^{p} i_{l}=i} \operatorname{Hom}_{K(R G)}\left(\mathbf{P}, \mathbf{P}\left[i_{l}\right]\right)\right) \otimes_{R} \operatorname{Hom}_{K\left(R \mathfrak{S}_{p}\right)}\left(\mathbf{Q}, \mathbf{Q}_{s}[j]\right)\right) \\
& \left.\simeq \bigoplus_{i+j=n} \operatorname{Hom}_{K(R G)}(\mathbf{P}, \mathbf{P}[i]) \otimes_{R} \operatorname{Hom}_{K\left(R C_{p}\right)}(\mathbf{Q}, \mathbf{Q}[j])\right),
\end{aligned}
$$

since $\operatorname{Hom}_{R}^{\bullet}(\mathbf{P}, \mathbf{P}) \simeq R$.

Similarly,

$$
\begin{gathered}
\operatorname{res}_{G \times C_{p}}^{G i \mathfrak{S}_{p}}\left(\operatorname{Hom}_{K\left(R G i \mathfrak{S}_{p}\right)}\left(X^{G \imath \mathfrak{S}_{p}} \otimes_{G \imath \mathfrak{S}_{p}} \mathbf{P}^{G \imath \mathfrak{S}_{p}}, X^{G l \mathfrak{S}_{p}} \otimes_{G l \mathfrak{S}_{p}} \mathbf{P}^{G l \mathfrak{S}_{p}} \otimes_{R} R_{s}^{(n)}[n]\right)\right) \\
\simeq \operatorname{res} s_{G \times C_{p}}^{G i \mathfrak{S}_{p}}\left(\bigoplus_{i+j=n}\left(\bigotimes_{\sum_{l=1}^{p} i_{l}=i} \operatorname{Hom}_{K(R G)}\left(X \otimes_{G} \mathbf{P}, X \otimes_{G} \mathbf{P}\left[i_{l}\right]\right)\right)\right. \\
\left.\simeq \otimes_{R} \operatorname{Hom}_{K\left(R \mathfrak{S}_{p}\right)}\left(\mathbf{Q}, \mathbf{Q}_{s}[j]\right)\right) \\
\simeq \bigoplus_{i+j=n} \operatorname{Hom}_{K(R G)}\left(X \otimes_{G} \mathbf{P}, X \otimes_{G} \mathbf{P}[i]\right) \otimes_{R} \operatorname{Hom}_{K\left(R C_{p}\right)}(\mathbf{Q}, \mathbf{Q}[j])
\end{gathered}
$$

by the same isomorphisms. This proves that the square $(*)$ is commutative.

It is now direct to see that the action of $X$ on the Ext-algebra commutes with mod $p$ Steenrod operations. In fact, the left square of the diagram

$$
\begin{aligned}
& H^{n}\left(G, \mathbb{F}_{p}\right) \stackrel{\text { orm }_{G}^{G \times C_{p}}}{\longrightarrow} \quad H^{p n}\left(G \times C_{p}, \mathbb{F}_{p}\right) \simeq \bigoplus_{i=0}^{n p} H^{i}\left(G, \mathbb{F}_{p}\right) \otimes_{\mathbb{F}_{p}} H^{n p-i}\left(C_{p}, \mathbb{F}_{p}\right) \\
& X \otimes-\downarrow \quad(X \otimes i d) \otimes_{G \times C_{p}}-\downarrow \quad \bigoplus_{i=0}^{n p}(X \otimes i d) \otimes-\downarrow \\
& H^{n}\left(G, \mathbb{F}_{p}\right) \stackrel{\operatorname{norm}_{G}^{G \times C_{p}}}{\longrightarrow} \quad H^{p n}\left(G \times C_{p}, \mathbb{F}_{p}\right) \simeq \bigoplus_{i=0}^{n p} H^{i}\left(G, \mathbb{F}_{p}\right) \otimes_{\mathbb{F}_{p}} H^{n p-i}\left(C_{p}, \mathbb{F}_{p}\right)
\end{aligned}
$$


is commutative, as is shown above. The right square is clearly commutative for $p=2$, since the cohomology of a cyclic group of order $2 \mathrm{~s} \mathbb{F}_{2}$ in each degree. For odd $p$ the right square is commutative up to a scalar, which is easily seen to be the identity.

The proof of the theorem is finished.

\section{Consequences}

Let $G$ be a finite group. As in [12] we denote by $H \operatorname{Splen}_{\mathbb{F}_{p}}(G)$ the subgroup of homotopy equivalence classes of splendid tilting complexes in $\operatorname{TrPi\mathbb {F}_{p}}\left(\mathbb{F}_{p} G\right)$ which in addition map the trivial $\mathbb{F}_{p} G$-module to an (in the derived category of $\mathbb{F}_{p} G$ modules) isomorphic copy.

By Theorem 1 the action of the mod $p$ Steenrod algebra $\mathcal{A}_{p}$ and the action of $H D_{\mathbb{F}_{p}}(G)$ on $H^{*}\left(G, \mathbb{F}_{p}\right)$ commute. Hence the cohomology ring $H^{*}\left(G, \mathbb{F}_{p}\right)$ is an $\mathcal{A}_{p}-H D_{\mathbb{F}_{p}}(G)$-bimodule.

Let $\mathfrak{U}_{p}$ be the category of unstable modules over the mod $p$ Steenrod algebra (cf. e.g., Schwartz [10]). Then, for any group $G$ we get a functor $\mathcal{D}_{p}(G)$ defined by

$$
H^{*}\left(G, \mathbb{F}_{p}\right) \otimes_{\mathbb{F}_{p} H D_{\mathbb{F}_{p}}(G)}-: \mathbb{F}_{p} H D_{\mathbb{F}_{p}}(G)-\bmod \longrightarrow \mathfrak{U}_{p}
$$

By the usual adjointness formula

$$
\begin{aligned}
& \operatorname{Hom}_{\mathbb{F}_{p}} \operatorname{HD}_{\mathbb{F}_{p}}(G) \\
& \quad \simeq \operatorname{Hom}_{\mathfrak{U}_{p}}\left(H^{*}\left(G, \mathbb{F}_{p}\right) \otimes_{\mathbb{F}_{p} H{\mathfrak{U}_{F}}_{p}(G)}\left(H^{*}\left(G, \mathbb{F}_{p}\right), ?\right)\right) \\
& \quad, ?
\end{aligned}
$$

as bifunctors $\mathbb{F}_{p} H D_{\mathbb{F}_{p}}(G)$-mod $\times \mathfrak{U}_{p} \longrightarrow \mathcal{E} n s$ the functor $\mathcal{D}_{p}(G)$ has a right adjoint $\mathcal{E}_{p}(G):=\operatorname{Hom}_{\mathfrak{U}_{p}}\left(H^{*}\left(G, \mathbb{F}_{p}\right),-\right)$.

Of course there is a dual version of the above defined by $\mathcal{E}_{p}^{H o m}(G)$ :

$$
-\otimes_{\mathcal{A}_{p}} H^{*}\left(G, \mathbb{F}_{p}\right): \mathfrak{U}_{p} \longrightarrow \bmod -\left(\mathbb{F}_{p} H D_{\mathbb{F}_{p}}(G)\right)
$$

which has a right adjoint $\mathcal{D}_{p}^{H o m}(G):=\operatorname{Hom}_{\mathbb{F}_{p} H D_{\mathbb{F}_{p}}(G)}\left(H^{*}\left(G, \mathbb{F}_{p}\right),-\right)$.

We get a system of functors

$$
\begin{array}{ll}
\mathcal{D}_{p}(G): & \mathbb{F}_{p} H D_{p}(G) \text {-mod } \longrightarrow \mathfrak{U}_{p}, \\
\mathcal{D}_{p}^{H o m}(G): & \mathbb{F}_{p} H D_{p}(G)-\bmod \longrightarrow \mathfrak{U}_{p}, \\
\mathcal{E}_{p}(G): & \mathbb{F}_{p} H D_{p}(G)-\bmod \longleftarrow \mathfrak{U}_{p}, \\
\mathcal{E}_{p}^{\otimes}(G): & \mathbb{F}_{p} H D_{p}(G)-\bmod \longleftarrow \mathfrak{U}_{p},
\end{array}
$$

where $\left(\mathcal{D}_{p}(G), \mathcal{E}_{p}(G)\right)$ and $\left(\mathcal{D}_{p}^{H o m}(G), \mathcal{E}_{p}^{\otimes}(G)\right)$ are adjoint pairs and where we abbreviate $H D_{p}(G)$ for $H D_{\mathbb{F}_{p}}(G)$.

First, not very astonishing, we are able to find the image of the free unstable modules $F(n)$ by $\mathcal{E}_{p}^{\otimes}(G)$. Recall that $F(n)$ are determined as representing object to the functor $M \mapsto M^{n}$ for any $n \in \mathbb{N}$ :

$$
\operatorname{Hom}_{\mathfrak{U}_{p}}(F(n),-) \simeq(-)^{n} .
$$

Lemma 4.1. $\mathcal{E}_{p}^{\otimes}(G)(F(n)) \simeq H^{*}(G)^{n}$ as $\mathbb{F}_{p} H D_{p}(G)$-modules.

Proof. We apply Yoneda's lemma to the equation

$$
\begin{aligned}
\operatorname{Hom}_{\mathbb{F}_{p} H D_{p}(G)}\left(F(n) \otimes_{\mathfrak{A}_{p}} H^{*}(G),-\right) & \simeq \operatorname{Hom}_{\mathfrak{U}_{p}}\left(F(n), \operatorname{Hom}_{H D_{p}(G)}\left(H^{*}(G),-\right)\right) \\
& \simeq \operatorname{Hom}_{\mathbb{F}_{p} H D_{p}(G)}\left(H^{*}(G),-\right)^{n} \\
& \simeq \operatorname{Hom}_{\mathbb{F}_{p} H D_{p}(G)}\left(H^{*}(G)^{n},-\right)
\end{aligned}
$$

to get the result. 
We shall study the compatibility with Lannes' $T$-functor (cf. e.g., [10]). For this, we observe that by functoriality, if $N$ is an $\mathcal{A}_{p}-H D_{k}(G)$-bimodule, so is $T_{V}(N)$.

Proposition 4.2. Let $V$ be an elementary abelian p-group and $M$ an $H D_{\mathbb{F}_{p}}(G)$ module. Then,

$$
\left(T_{V} \circ\left(\mathcal{D}_{p}(G)\right)\right) \simeq T_{V}\left(H^{*}(G)\right) \otimes_{\mathbb{F}_{p} H D_{\mathbb{F}_{p}}(G)}-.
$$

Proof. We use the different adjointness formulas:

$$
\begin{aligned}
\operatorname{Hom}_{\mathfrak{U}_{p}}\left(T_{V}\left(\mathcal{D}_{p}(G) M\right),-\right) & \simeq \operatorname{Hom}_{\mathfrak{U}_{p}}\left(\mathcal{D}_{p}(G)(M), H^{*}(V) \otimes_{\mathbb{F}_{p}}-\right) \\
& \simeq \operatorname{Hom}_{H D_{p}(G)}\left(M, \operatorname{Hom}_{\mathfrak{U}_{p}}\left(H^{*}(G), H^{*}(V) \otimes_{\mathbb{F}_{p}}-\right)\right) \\
& \simeq \operatorname{Hom}_{H D_{p}(G)}\left(M, \operatorname{Hom}_{\mathfrak{U}_{p}}\left(T_{V}\left(H^{*}(G),-\right)\right)\right. \\
& \simeq \operatorname{Hom}_{\mathfrak{U}_{p}}\left(T_{V}\left(H^{*}(G)\right) \otimes_{H D_{p}(G)} M,-\right)
\end{aligned}
$$

The rest follows by Yoneda's lemma.

We shall elaborate more on Proposition 4.2. As stated in Henn, Lannes and Schwartz 4, Theorem 5.2] (see [3] for a proof), Lannes found that

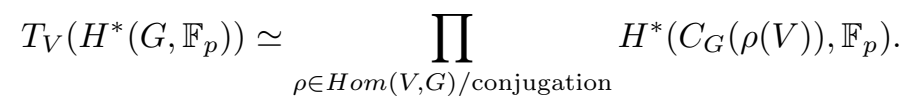

Let us recall how the mapping is constructed. For any fixed $\rho \in \operatorname{Hom}(V, G)$ one gets

$$
\begin{aligned}
V \times C_{G}(i m \rho) & \stackrel{\mu_{\phi}}{\longrightarrow} C_{G}(i m \rho) \hookrightarrow G, \\
(v, c) & \mapsto \rho(v) c .
\end{aligned}
$$

Taking cohomology, this induces a mapping

$$
H^{*}\left(G, \mathbb{F}_{p}\right) \stackrel{r e S_{G}^{G}(i m \rho)}{\longrightarrow} H^{*}\left(C_{G}(i m \rho), \mathbb{F}_{p}\right) \longrightarrow H^{*}\left(V, \mathbb{F}_{p}\right) \otimes_{\mathbb{F}_{p}} H^{*}\left(C_{G}(i m \rho), \mathbb{F}_{p}\right) .
$$

Now, $T_{V}$ is left adjoint to $H^{*}\left(V, \mathbb{F}_{p}\right) \otimes_{\mathbb{F}_{p}}-$, and therefore, this mapping induces a mapping

$$
\left.T_{V}\left(H^{*}\left(G, \mathbb{F}_{p}\right)\right) \stackrel{\operatorname{ad}\left(\mu_{\phi}^{*}\right. \text { ores }}{\longrightarrow}\right) H^{*}\left(C_{G}(i m \rho)\right) .
$$

Taking the product of these mappings gives the required isomorphism.

In [12] we obtained that the Brauer construction with respect to the diagonal of a $p$-subgroup of $G$ in $G \times G$ yields a group homomorphism

$$
\text { SplenPic } \mathbb{F}_{\mathbb{F}_{p}}(G) \longrightarrow \operatorname{SplenPic}_{\mathbb{F}_{p}}\left(C_{G}(Q)\right) \text {. }
$$

In [12] we were not able to prove that under this homomorphism the property of fixing the trivial module is preserved. Nevertheless, we conjecture that this is true.

Conjecture 4.3. The mapping SplenPic $i_{\mathbb{F}_{p}}(G) \longrightarrow \operatorname{SplenP}_{c_{\mathbb{F}_{p}}}\left(C_{G}(Q)\right)$ induces a mapping HSplen $\mathbb{F}_{p}(G) \longrightarrow H$ Splen $_{\mathbb{F}_{p}}\left(C_{G}(Q)\right)$.

Supposing Conjecture 4.3 is true, we shall show that the decomposition

$$
T_{V}\left(H^{*}\left(G, \mathbb{F}_{p}\right)\right)=\prod_{\rho} H^{*}\left(C_{G}(i m \rho), \mathbb{F}_{p}\right)
$$

is compatible under the action of $H \operatorname{Splen}_{\mathbb{F}_{p}}(G)$ from the right.

Actually, the mapping

$$
H \operatorname{Splen}_{\mathbb{F}_{p}}(G) \rightarrow H \operatorname{Splen}_{\mathbb{F}_{p}}\left(C_{G}(Q)\right)
$$


then gives an $\mathbb{F}_{p} H S p l e n_{\mathbb{F}_{p}}(G)$-module structure on $H^{*}\left(C_{G}(i m \rho), \mathbb{F}_{p}\right)$ for any $\rho \in \operatorname{Hom}(V, G)$.

Proposition 4.4. Suppose that Conjecture 4.3 is true. Then, the mappings above induce a decomposition

$$
T_{V}\left(H^{*}\left(G, \mathbb{F}_{p}\right)\right)=\prod_{\rho} H^{*}\left(C_{G}(i m \rho), \mathbb{F}_{p}\right)
$$

as $\mathcal{A}_{p}-\mathbb{F}_{p} H$ Splen $\mathbb{F}_{p}(G)$-bimodules.

Proof. Let $X$ be a two-sided tilting complex in $H \operatorname{Splen}_{\mathbb{F}_{p}}(G)$. In 12 it is proved that the diagram

$$
\begin{aligned}
& H^{*}\left(G, \mathbb{F}_{p}\right) \stackrel{\operatorname{res}_{C_{G}(i m \rho)}^{G}}{\longrightarrow} H^{*}\left(C_{G}(i m \rho), \mathbb{F}_{p}\right) \\
& X \otimes_{G}-\downarrow \\
& H^{*}\left(G, \mathbb{F}_{p}\right) \stackrel{\operatorname{res}_{C_{G}(i m \rho)}^{G}}{\longrightarrow} H^{*}\left(C_{G}(i m \rho), \mathbb{F}_{p}\right)
\end{aligned}
$$

is commutative.

Now, $V$ is abelian. So, $i m \rho \subseteq C_{G}(i m \rho)$ for any $\rho \in \operatorname{Hom}(V, G)$. Let $\mathbf{Q}$ be a free resolution of the trivial $\mathbb{F}_{p} V$-module and let $\mathbf{P}$ be a projective resolution of the trivial $\mathbb{F}_{p} C_{G}(i m \rho)$-module. Then, $\mathbf{Q} \otimes_{\mathbb{F}_{p}} \mathbf{P}$ is a projective resolution of the trivial $\mathbb{F}_{p}\left(V \times C_{G}(i m \rho)\right)$-module. In order to describe the mapping $H^{*}\left(C_{G}(i m \rho), \mathbb{F}_{p}\right) \longrightarrow$ $H^{*}\left(V, \mathbb{F}_{p}\right) \otimes_{\mathbb{F}_{p}} H^{*}\left(C_{G}(i m \rho), \mathbb{F}_{p}\right)$ we have to determine a mapping $\tau$ which makes the diagram of $\mathbb{F}_{p}\left(V \times C_{G}(i m \rho)\right)$-modules

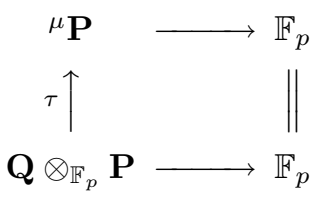

commutative. Since $\mathbf{Q}$ is a free resolution, and since $i m \rho \subseteq C_{G}(i m \rho)$, the resolution $\mathbf{P}$ is a $C_{G}(\mathrm{im} \rho)$-module, and the mapping $\tau$ can simply be chosen to be multiplication via $\rho$. It is obvious that this makes the diagram commutative.

Denote for the moment $X^{\prime}:=X\left(\Delta\left(C_{G}(i m \rho)\right)\right)$. Then, choosing these particular resolutions and mappings, it is easily seen that

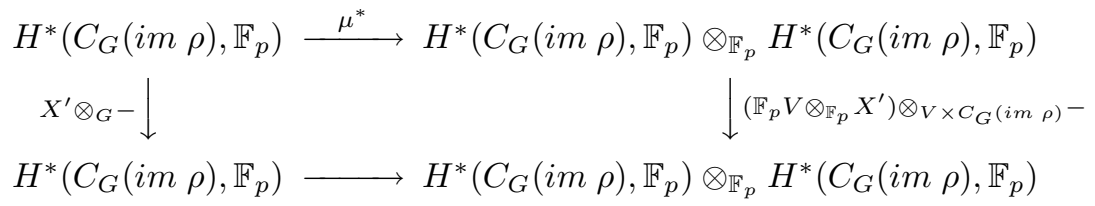

is commutative.

Now, it is sufficient to consider the adjoint mappings to the above. These yield commutative diagrams

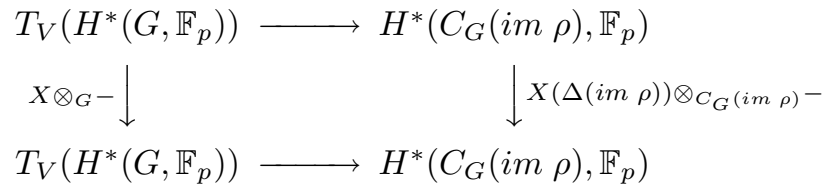

by the functoriality of the adjointness formulas. This finishes the proof. 
Remark 4.5. If Conjecture 4.3 is not true, Proposition 4.4 remains true for the action of

$$
\bigcap_{\rho \in H o m(V, G)}\left(-\left(\Delta\left(C_{G}(i m \rho)\right)\right)\right)^{-1}\left(H \operatorname{Splen}_{\mathbb{F}_{p}}\left(C_{G}(i m \rho)\right)\right)
$$

on $T_{V}\left(H^{*}\left(G, \mathbb{F}_{p}\right)\right)$.

Remark 4.6. Observe that we really needed to have splendid equivalences in the proof of Proposition 4.4. Moreover, Lemma 4.2 does not use any hypothesis on the nature of the equivalences. Since the decomposition of $T_{V}\left(H^{*}\left(G, \mathbb{F}_{p}\right)\right)$ as an unstable module holds in general, one would like to have an interpretation of this decomposition as a module over the bigger group of self-equivalences. This seems to include a generalization of [12].

\section{REFERENCES}

1. David Benson, Representations and cohomology II, Cambridge 1991. MR 93g:20099

2. Charles W. Curtis and Irving Reiner, Methods of Representation Theory, Vol I John Wiley and Sons 1981. MR 82i:20001

3. Hans-Werner Henn, Cohomology of groups and unstable modules over the Steenrod algebra, Advanced course on classifying spaces and cohomology of groups, Centre de Recerca Matemàtica Bellaterra (Spain). http://www-irma.u-strasbg.fr/ ${ }^{\sim}$ henn/notes.ps

4. Hans-Werner Henn, Jean Lannes and Lionel Schwartz, Locations of unstable A-modules and equivariant mod p cohomology, Math. Ann. 301 (1995) 23-68. MR 95k:55036

5. Steffen König and Alexander Zimmermann, Derived equivalences for group rings, (with contributions by Bernhard Keller, Markus Linckelmann, Jeremy Rickard and Raphaël Rouquier) Springer LNM 1685 (1998) MR 2000g:16018

6. Andrei Marcus, On equivalences between blocks of group algebras: Reduction to the simple components, Journal of Algebra 184 (1996) 372-396. MR 97m:20012

7. Jeremy Rickard, Morita theory for derived categories, J. London Math. Soc. 39 (1989) 436456. MR 91b:18012

8. Jeremy Rickard, Splendid equivalences: derived categories and permutation modules, Proc. London Math. Soc. 72 (1996) 331-358. MR 97b:20011

9. Raphaël Rouquier and Alexander Zimmermann, A Picard group for derived module categories, accepted by Proc. London Math. Soc.

10. Lionel Schwartz, Unstable modules over the Steenrod algebra and Sullivan's fixed point set conjecture, Chicago Lectures in Mathematics, Chicago University Press 1994. MR 95d:55017

11. Alexander Zimmermann, Auto-equivalences of derived categories acting on cohomology, to appear in Archiv der Mathematik.

12. Alexander Zimmermann, Cohomology of groups and splendid equivalences of derived categories, Math. Proc. Cambridge Phil. Soc. 131 (2001) 459-472.

Faculté de Mathématiques et CNRS (LAMFA FRE 2270), Université de Picardie, 33 Rue St Leu, 80039 Amiens Cedex, France

E-mail address: Alexander.Zimmermann@u-picardie.fr

URL: http://www.mathinfo.u-picardie.fr/alex/azim.html 